\title{
Flora da Bahia: Zygophyllaceae
}

\section{Uiara Catharina Soares e Silva ${ }^{1 *}$, Reyjane Patrícia de Oliveira ${ }^{1, a}$, Raymond Mervyn Harley ${ }^{2, b}$, Ana Maria Giulietti ${ }^{1,2, c}$}

${ }^{1}$ Programa de Pós-Graduação em Botânica, Universidade Estadual de Feira de Santana, Bahia, Brasil.

2 Royal Botanic Gardens, Kew, Reino Unido.

Resumo - É apresentado o levantamento florístico de Zygophyllaceae da Bahia, Brasil, como contribuição ao conhecimento da Flora do Estado. São reconhecidas as espécies Kallstroemia tribuloides e Tribulus terrestris. São apresentadas chave de identificação, descrições e ilustrações, além de comentários gerais sobre os táxons.

Palavras-chave adicionais: Brasil, florística, Kallstroemia, Tribulus.

Abstract (Flora of Bahia: Zygophyllaceae) - This account of the Zygophyllaceae is a further contribution to the ongoing Flora of Bahia project. Kallstroemia tribuloides and Tribulus terrestris are recognized for the state of Bahia, Brazil. A key to species is provided together with descriptions, illustrations, and general notes for the taxa.

Additional key words: Brazil, floristics, Kallstroemia, Tribulus.

\section{ZYGOPHYLLACEAE}

Ervas, arbustos ou árvores, anuais ou perenes. Folhas opostas ou raramente alternas, geralmente compostas, paripinadas, pecioladas a subsésseis; folíolos inteiros ou ocasionalmente lobados; estípulas livres, persistentes ou raramente caducas. Inflorescências axilares ou terminais, racemosas ou fasciculadas, frequentemente reduzidas a uma única flor. Flores geralmente vistosas, bissexuadas, actinomorfas, diclamídeas; (4)5(6)-meras; sépalas livres ou raramente conatas na base, persistentes ou decíduas; pétalas livres ou 3 pétalas unidas e 2 livres; prefloração valvar ou imbricada; disco glandular extraestaminal e/ou intraestaminal geralmente presente e conspícuo; estames (5)6-10(-12); anteras introrsas, 2-tecas, rimosas; gineceu (2-)5-carpelar, sincárpico; ovário súpero, (2-)5(-12)-locular; estilete 1 , às vezes muito curto; estigma 1 a numerosos, capitado ou lobado; óvulos (1)2 a numerosos em cada lóculo; placentação axilar ou raramente basal. Fruto cápsula (2-)5-lobada, loculicida, septicida ou esquizocarpo, separando-se longitudinalmente em 5-10(-12) mericarpos rígidos, tuberculados a espinescentes ou alados; sementes com endosperma rígido, oleoso ou ausente; embrião com cotilédones lineares ou oblongos.

A família inclui ca. 24 gêneros e 275 espécies, sendo amplamente distribuída, principalmente nas regiões áridas dos trópicos e subtrópicos (Heywood et al. 2007). Dada a presença de alcaloides e/ou resina, muitas espécies desta família têm sido utilizadas na medicina popular em diversos países, bem como na

\footnotetext{
*Autora para correspondência: uiara_catharina@yahoo.com.br;

arpolliveira@yahoo.com; br.harley@kew.org;

canagiulietti@hotmail.com

Editor responsável: Pedro Fiaschi

Submetido: 20 mar. 2014; aceito: 23 jul. 2014

Publicação eletrônica: 15 ago. 2014; versão final: 22 ago 2014
}

conservação de alimentos (Maciel \& Alves 2009). Para o Brasil, Engler (1872) indicou dois gêneros, Kallstroemia Scop. e Tribulus L., com duas espécies cada. Ele se referiu ainda a outras espécies americanas que poderiam ocorrer no Brasil, como K. maxima Torr. \& Gray. Já Souza \& Lorenzi (2012) indicaram, além dos dois gêneros já citados, também Bulnesia Gay, sendo B. sarmientoi Lorentz ex Griseb. a única espécie arbórea de Zygophyllaceae nativa no Brasil. Sobral (2014), por sua vez, considerou apenas a ocorrência de Kallstroemia para o Brasil, com duas espécies: $K$. maxima (L.) Hook. \& Arn. e K. tribuloides (Mart.) Steud.; a ocorrência da primeira espécie, no entanto, deve ser reavaliada (veja os comentários do gênero).

Para o estado da Bahia, são aqui reconhecidos os gêneros Kallstroemia e Tribulus, com uma espécie cada. Guaiacum officinale L., conhecida popularmente como pau-santo, é exótica, cultivada em algumas áreas da Bahia, e não foi incluída no presente trabalho.

\section{Chave para as espécies}

1. Frutos tuberculados, separando-se na maturidade em 8-12 mericarpos; parte central do receptáculo persistente 1.1. Kallstroemia tribuloides

1'. Frutos espinescentes, separando-se na maturidade em 5 mericarpos; parte central do receptáculo caindo com os mericarpos .

\subsection{Tribulus terrestris}

\section{Kallstroemia Scop.}

Ervas anuais; ramos herbáceos, prostrados a decumbentes. Folhas opostas, paripinadas; folíolos inteiros, elípticos a oblongos ou obovados, pubescentes, (2-)4-6 pares, um folíolo de cada par alternadamente menor ou abortado; pecíolo curto; estípulas interpeciolares foliáceas, estreitas a amplamente falcadas, acuminadas. Inflorescências reduzidas a uma única flor, axilares ou pseudoaxilares. 
Flores pedunculadas; sépalas 5, livres, inteiras, persistentes; pétalas 5, livres, obovadas ou obcordadas, brancas a alaranjadas, decíduas; estames 10-12; glândulas intraestaminais presentes; filetes filiformes; anteras geralmente globosas; ovário séssil, glabro a pubescente, 8-12-locular, 1 óvulo por lóculo, pêndulo; estilete simples, persistente, formando um rostelo quando em fruto; estigma capitado ou clavado. Fruto esquizocarpo, separando-se na maturidade geralmente em 10 mericarpos; semente 1 por mericarpo, oblonga a oval, testa membranácea, pouco endosperma.

O gênero possui 16 espécies que se distribuem nas regiões áridas das Américas, dos Estados Unidos à Argentina (Porter 1969, 2001; Heywood et al. 2007). Como mencionado anteriormente, Sobral (2014) indicou Kallstroemia maxima e $K$. tribuloides para o Brasil, ambas representadas na Bahia. A ocorrência de K. maxima está baseada em Harley 16250, depositado no herbário MO. Apesar de não termos examinado este material, as três duplicatas dessa coleta depositadas no herbário K se referem a $K$. tribuloides. Como proposto por Porter (1969), essas duas espécies podem ser diferenciadas facilmente pelo formato e posicionamento dos folíolos: $K$. tribuloides tem os folíolos elípticos e os maiores situam-se na porção mediana da folha, enquanto em $K$. maxima, os folíolos são obovados e os maiores situam-se na porção apical da folha. Além disso, K. tribuloides ocorre na América do Sul, incluindo Bolívia, Argentina e Brasil, enquanto $K$. maxima ocorre na costa sudoeste dos Estados Unidos, México, Caribe e norte da América do Sul. Dessa maneira, não incluímos $K$. maxima neste tratamento, e a ocorrência dessa espécie no Brasil deve ser reavaliada.

\subsection{Kallstroemia tribuloides (Mart.) Steud., Nomencl.} Bot. 1: 844. 1840.

Figuras $1 \mathrm{~A}-\mathrm{C}$ e 2.

Nome popular: rabo-de-calango.

Ervas, prostradas. Folhas 1,6-4,9 × 0,9-2,6 cm; estípulas verdes, pubescentes, persistentes, falcadas, acuminadas; folíolos $0,8-1,9 \times 0,2-0,8 \mathrm{~cm}, 3-6$ pares, pubescentes na face abaxial, elípticos, papiráceos. Pedúnculo 1-1,3 cm compr. Flores isoladas, axilares, 1,5-4,3 cm diâm.; sépalas elípticas, persistentes; pétalas obovadas; estames 10, inseridos na base do disco; ovário 10-locular, glabro; estilete com porção distal dilatada, lobado. Frutos tuberculados, indeiscentes, reticulados, sem espinhos laterais; mericarpos 10, com a parte central do receptáculo persistente; semente com testa membranácea.

América do Sul tropical, em vegetação de caatinga (Maciel \& Alves 2009). A8, B8, B9, C7, C8, D6, D7, D9, E5, E7, E8, F4. Floresce e frutifica de novembro a julho.

Material examinado - Abaré, beira do rio São Francisco, 0843'46”S, 3904'35”W, 321 m s.n.m., 1 nov. 2009 (fl., fr.), E. Melo et al. 7052 (HUEFS); Bendengó, margem da BR-116, 10 km da cidade em direção a Euclides da Cunha, 1001'58'S, 3909'W, 445 m s.n.m., 21 fev. 2000 (fl., fr.), A.M. Giulietti \& R.M. Harley 1720 (HUEFS); Bom Jesus da Lapa, morro acima da igreja, no centro da cidade, $13^{\circ} 15$ ' $32^{\prime}$ 'S, $43^{\circ} 25^{\prime} 11^{\prime \prime} \mathrm{W}, 640 \mathrm{~m}$ s.n.m., $10 \mathrm{fev}$. 2000 (fl., fr.), L.P. Queiroz et al. 5816 (HUEFS); Boquira, arredores da cidade, 19 jan. 1997 (fl., fr.), G. Hatschbach et al. 65985 (CEPEC, MBM); Canudos, em frente ao hotel Brasil, na rua, 09 53'47'S, 3901'47'W, 373 m s.n.m., 19 fev. 2004 (fl., fr.), R.M. Harley et al. 54905 (HUEFS); Cipó, 13 maio 1981 (fr.), L.M.C. Gonçalves 50 (HRB, HUEFS); Glória, ca. 4 km de Olhos d'Água de Souza, 09²0'37'S, 38¹8'57’W, 373 m s.n.m., 26 abr. 2001 (fl., fr.), L.P. Queiroz et al. 6539 (HUEFS); Iaçu, Lage Preta, 12 50'21"S, 39 58'15”W, 360 m s.n.m., fev. 2005 (fl.), F. França et al. 5145 (HUEFS); Itiúba, ca. $10 \mathrm{~km}$ da cidade, 10³9'28”S, $39^{\circ} 44^{\prime} 16^{\prime \prime} \mathrm{W}, 375$ m s.n.m., 28 jan. 2002 (fl., fr.), J.G. Nascimento \& T.S. Nunes 33 (HUEFS); Jaguarari, base da Crista, próximo ao rio, $10^{\circ} 06^{\prime} 12^{\prime \prime S}, 40^{\circ} 13^{\prime} 47^{\prime \prime} \mathrm{W}, 560 \mathrm{~m}$ s.n.m., 13 abr. 2006 (fl., fr.), R.F. Souza-Silva et al. 206 (HUEFS); Mairi, 29 mar. 1985 (fl., fr.), E.L.P.G. Oliveira 664 (EBAPA, HUEFS); Morro do Chapéu, 33 km na estrada para América Dourada, 13 jan. 1991 (fl., fr.), N.P. Taylor et al. 1409 (K); Paulo Afonso, 17 maio 1973 (fl., fr.), A.P. Duarte 14177 (K); São Gabriel, estrada para Irecê, $11^{\circ} 01^{\text {'S, }}$, 41 $39^{\prime}$ 'W, 15 abr. 2009 (fl., fr.), R.F. Machado et al. 258 (HUEFS); Senhor do Bonfim, Campus da UNEB, 7 nov. 2005 (fl., fr.), $V$. Barreto 154 (HUEFS, HUNEB).

Material adicional examinado - Alagoas, G. Gardner 1264 (K, 3 exsicatas); Piauí, Boa Esperança, G. Gardner 2084 (K).

Kallstroemia tribuloides distingue-se de Tribulus terrestris L., que também ocorre na Bahia, por apresentar frutos esquizocárpicos, geralmente com 10 mericarpos unidos no ápice pela porção distal do estilete, permanecendo a coluna central após a separação dos mericarpos.

\section{Tribulus L.}

Ervas, anuais ou ocasionalmente perenes; ramos herbáceos, prostrados ou procumbentes. Folhas opostas, paripinadas; folíolos inteiros, oblongos a ovaloblongos ou elípticos, assimétricos na base, 3-10 pares, um folíolo de cada par alternadamente menor ou abortado; pecíolo muito curto; estípulas verdes, interpeciolares, foliáceas. Inflorescências reduzidas a uma única flor axilar. Flores pedunculadas; sépalas 5 , livres, inteiras, lanceoladas, pubescentes na face adaxial, decíduas ou raramente persistentes; pétalas 5, livres, obovadas, amarelas ou raramente brancas, decíduas; estames 5-10, inseridos na base do disco, glândulas intraestaminais presentes, livres ou conatas abaixo da base do ovário; filetes filiformes; anteras oblongas a cordadas; estilete alongado, estigma 5lobado; ovário séssil, glabro a pubescente, 5-locular, 2-5(-10) óvulos por lóculo; placentação axilar. Fruto esquizocarpo, mericarpos rígidos, tuberculados ou espinescentes, separando-se na maturidade em 5 mericarpos, 1 semente por mericarpo, oblongoobovada, testa membranácea, endosperma ausente. 


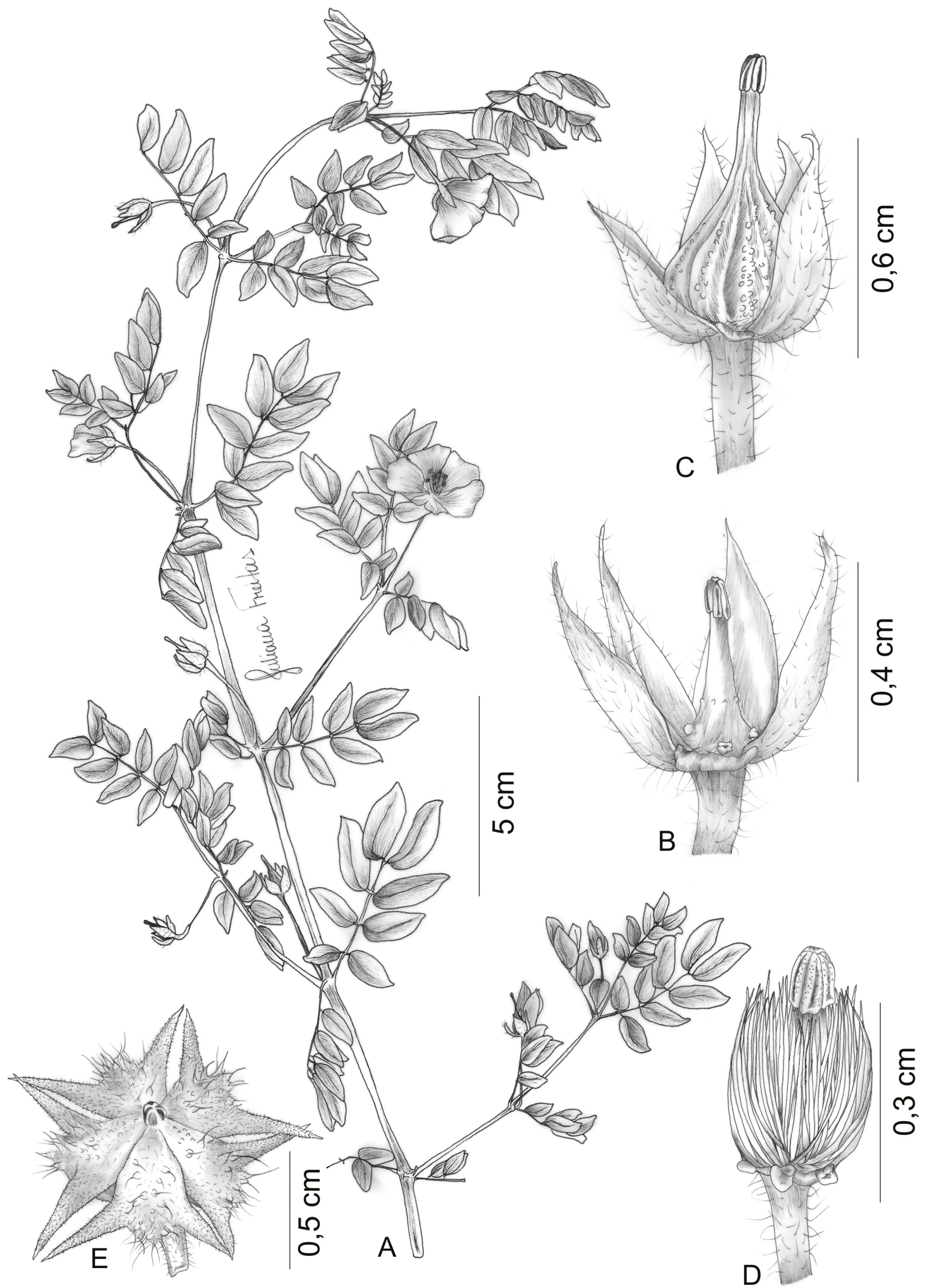

Figura 1. A-C. Kallstroemia tribuloides: A- ramo com flores e frutos; B- gineceu com sépalas persistentes e glândulas intraestaminais; Cfruto em vista lateral, com cálice persistente (uma sépala removida) D, E. Tribulus terrestris: D- gineceu envolto por tricomas, glândulas intraestaminais evidentes. E- fruto em vista frontal (A-C- Queiroz 5816; D, E- Nascimento 60). 
O gênero é representado por cerca de 25 espécies altamente polimórficas (Varghese et al. 2006). Nativo dos Paleotrópicos, foi introduzido e naturalizado no Novo Mundo (Aymard \& Cuello 2005). A maioria das espécies ocupa áreas secas, incluindo hábitats perturbados. Os mericarpos espinescentes de muitas espécies fornecem um mecanismo ideal para a sua disseminação (Porter 1972). No Brasil, inclusive no estado da Bahia, ocorre apenas Tribulus terrestris, em áreas de caatinga.

\subsection{Tribulus terrestris L., Sp. Pl. 1: 387. 1753.}

Figuras 1D-E e 2.

Nome popular: cabeça-de-touro, videira-da-punctura.

Ervas, prostradas. Folhas 1,2-4,8 × 1,2-2,3 cm; estípulas caducas, pubescentes, falcadas; folíolos 0,5 $1,4 \times 0,2-0,5 \mathrm{~cm}, 3-6$ pares, glabros na face adaxial, pubescentes na abaxial, oblongo-obovados. Pedúnculo ca. $0,3 \mathrm{~cm}$ compr. Flores isoladas, axilares, ca. $1 \mathrm{~cm}$ diâm.; sépalas oblongas a lanceoladas, caducas; pétalas espatuladas; estames 5, inseridos na base do disco; ovário pubescente, 3 ou 4 óvulos por lóculo; estilete sem porção distal dilatada; estigma terminal, lobado. Frutos espinescentes, indeiscentes, com 2 espinhos laterais em cada mericarpo, bico ausente; semente com testa membranácea.

Nativa da região Mediterrânea, provavelmente do nordeste da África, e bastante difundida nos trópicos (Porter 1972), apresenta-se como erva ruderal das caatingas brasileiras (Souza \& Lorenzi 2012), ocorrendo de maneira subespontânea. B5, B6, B7, B8, B9, C8, C9, D6, D7, D9, E7, F4, G4, G5. Floresce e frutifica de novembro a abril.

Material examinado - Bela Vista, $10^{\circ} 39^{\prime}$ 'S, $39^{\circ}$ 44”'W, 30 mar 2004 (fl., fr.), M.V. Moraes 674 (HUEFS); Bom Jesus da Lapa,

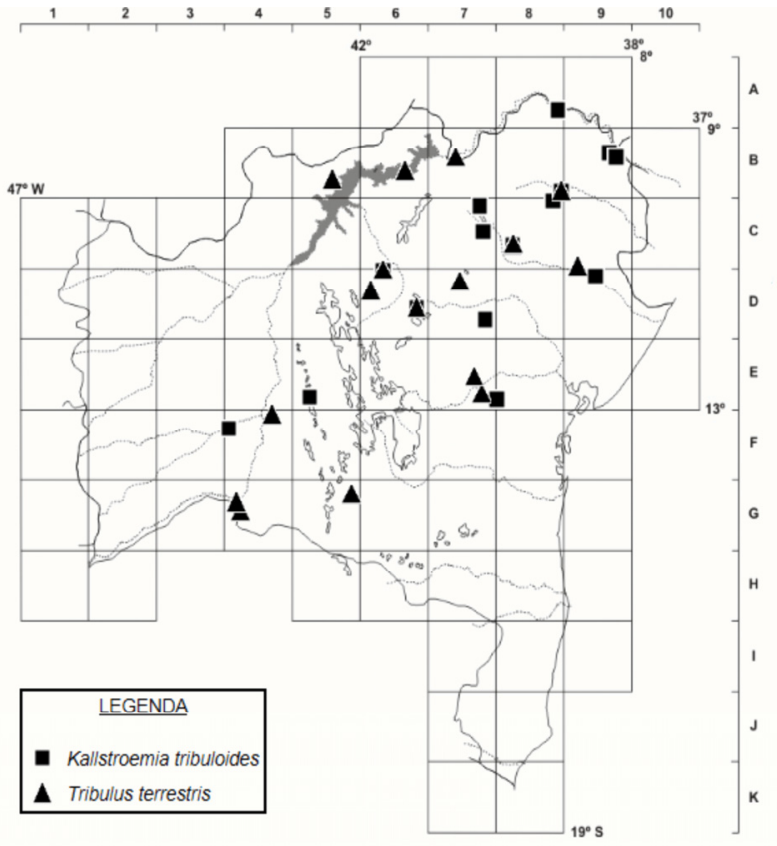

Figura 2. Mapa de distribuição de Kallstroemia tribuloides e Tribulus terrestris no estado da Bahia.

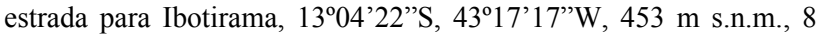
fev. 2000 (fl., fr.), L.P. Queiroz et al. 5730 (HUEFS); Canudos, em frente ao Hotel Brasil, 0953'47'S, 39 01'47'W, 373 m s.n.m., 19 fev. 2004, R.M. Harley et al. 54904 (HUEFS); Carinhanha, ca. de $1 \mathrm{~km}$ do rio Carinhanha, $14^{\circ} 18^{\prime} 41^{\prime \prime} \mathrm{S}, 43^{\circ} 48^{\prime} 32^{\prime \prime} \mathrm{W}, 462 \mathrm{~m}$ s.n.m., 28 dez. 2007 (fl., fr.), M.L. Guedes \& F.S. Gomes 14046 (ALCB, HUEFS); Casa Nova, fazenda Santarém, sítio Morrinho, área

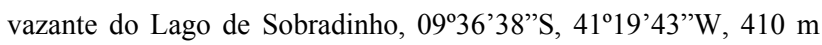
s.n.m., 10 out. 2004 (fl., fr.), L.P. Queiroz et al. 9660; Iaçu, $12^{\circ} 46^{\prime} 34^{\prime \prime}$, $40^{\circ} 11^{\prime} 58^{\prime \prime}$ W, 270 m s.n.m., 17 mar. 2004 (fl., fr.), L.P. Queiroz et al. 9166 (HUEFS); Irecê, saída do Lapão para Américo Dourado, fazenda Jequitibá, 2 mar. 2004 (fl., fr.), L.A. Mattos-Silva \& B.R. Santos 5069 (CEPEC, HUEFS); Itaberaba, BR-242 ca. 39 km da cidade, 12³0'32”S, 3956'41”W, 300 m s.n.m., 9 fev. 1992 (fl., fr.), L.P. Queiroz \& R.M. Harley 2605 (HUEFS, K); Itiúba, Rômulo Campos, Lago do Jacurici, área do DNOCS, 10³9'20"S, 39 44"16"W, 355 m s.n.m., 19 jan. 2002, J.G. Nascimento \& T.S. Nunes 60 (HUEFS); Jacobina, terreno baldio no Junco, 6 dez. 1998 (fl., fr.), M.V. Moraes 134 (CEPEC, HUEFS); Juazeiro, $7 \mathrm{~km}$ ao sul da cidade, 09²5'S, 40³5'W, 23 jan. 1993 (fl., fr.), W. Thomas et al. s.n. (CEPEC 57679); Lagoa Real, 16 jan. 1997 (fl., fr.), G. Hatschbach et al. 65822 (CEPEC, MBM); Malhada, rodovia para o distrito de Cana Brava, 14027'9"S, 43045'12"W, 500 m s.n.m., 2 abr. 2001 (fr.), J.G. Jardim et al. 3437 (CEPEC, HUEFS); Morro

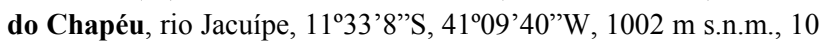
nov. 2007 (fl., fr.), F.B.L. Silva et al. 35 (HUEFS); Remanso, 09'44'17'S, 42²3'49”'W, 28 fev. 2000 (fl., fr.), A. Nascimento et al. 246 (ALCB, HUEFS, K); São Gabriel, estrada para Irecê, $11^{\circ} 1$ 'S, 4139'W, 15 abr. 2009 (fl., fr.), R.F. Machado et al. 259 (HUEFS); Tucano, Caldas do Jorro, Parque das Águas, 22 mar. 1992 (fl., fr.), A.M. Carvalho et al. 3886 (ALCB, CEPEC, HRB).

Reconhecida especialmente pelos frutos espinescentes com dois espinhos em cada mericarpo.

\section{AGRADECIMENTOS}

À curadoria dos herbários visitados, pelo acesso às coleções, e à Juliana Gomes Freitas, pelas ilustrações. À FAPESB e ao CNPq pelo apoio financeiro ao projeto Flora da Bahia. UCSS agradece à CAPES, pela bolsa de doutorado (PNADB). RPO e AMG agradecem ao $\mathrm{CNPq}$, pelas bolsas de produtividade em pesquisa (PQ2 e PQI-A, respectivamente).

\section{REFERÊNCIAS}

Aymard, C.G. \& Cuello, A.N. 2005. Zygophyllaceae. In: P.E. Berry, K. Yatskievych \& B. Holst (eds), Flora of the Venezuelan Guayana (Rutaceae-Zygophyllaceae). Vol. 9. Missouri Botanical Garden Press, Saint Louis, p. 580-584.

Engler, H.G.A. 1872. Zygophyllaceae. In: C.F. Martius \& A.W. Eichler (eds), Flora Brasiliensis. Vol. 12, pars 2. Frid. Fleischer, Leipizig, p. 66-74.

Heywood, V.H.; Brummitt, R.K.; Culham, A. \& Seberg, O. 2007. Flowering Plant Families of the World. Firefly Books, Ontario.

Maciel, J.R. \& Alves, M. 2009. Zygophyllaceae. In: M. Alves, M.F. Araújo, J.R. Maciel \& S. Martins (eds), Flora de 
Mirandiba. Associação Plantas do Nordeste, Recife, p. 353 355.

Porter, D.M. 1969. The genus Kallstroemia. Contributions from the Gray Herbarium of Harvard University 198: 41-153.

Porter, D.M. 1972. The genera of Zygophyllaceae in the southeastern United States. Journal of the Arnold Arboretum 53: 531-552.

Porter, D.M. 2001. Zygophyllaceae. In: W.D. Stevens \& C.U. Ulloa (eds), Flora de Nicaragua: Angiospermas (Pandanaceae - Zygophyllaceae). 85. Tomo III. Missouri Botanical Garden Press, Saint Louis, p. 2555-2556.
Sobral, M. 2014. Zygophyllaceae. In: Lista de Espécies da Flora do Brasil. Jardim Botânico do Rio de Janeiro. Disponível em $<$ http://floradobrasil.jbrj.gov.br/2012/FB000254>; acesso em 18 mar. 2014.

Souza, V.C. \& Lorenzi, H. 2012. Botânica Sistemática - guia ilustrado para identificação das famílias de fanerógamas nativas e exóticas do Brasil, baseado em APG III. 3 ed. Instituto Plantarum, Nova Odessa.

Varghese, M.; Yadav, S.S. \& Thomas, J. 2006. Taxonomic status of some of Tribulus species in Indian Subcontinent. Saudi Journal of Biological Sciences 13(1): 7-12.

\section{LISTA DE EXSICATAS}

Amaral-Santos, A. 2434 (2); Arbo, M.M. 7305 (1); Bandeira, F.P. 190 (1); Barreto, V. 154 (1); Carvalho, A.M. 3886 (2); CarvalhoSobrinho, J.G. 507 (2); Correia, C. 459 (2); Duarte, A.P. 14177 (1); França, F. 5145 (1); Gardner, G. 1264, 2084 (1); Giulietti, A.M. 1720 (1); Gonçalves, L.M.C. 50 (1); Guedes, M.L. 14046 (2); Harley, R.M. 16250, 16457 (1), 54904 (2), 54905 (1); Hatschbach, G. 61960, 65822 (2), 65985 (1); Jardim, J.G. 3437 (2); Machado, R.F. 258 (1), 259 (2); Mattos-Silva, L.A. 5069 (2); Melo, E. 7052 (1); Moraes, M.V. 134, 674 (2); Nascimento, A. 246 (2); Nascimento, J.G. 33 (1), 60 (2); Oliveira, E.L.P.G. 664 (1); Paula-Souza, J. 5441 (2); Queiroz, L.P. 2605 (2), 5730 (2), 5816, 6539 (1), 9166, 9660 (2); Silva, F.B.L. 35 (2); Souza Silva, R.F. 206 (1); Taylor N.P. 1409 (1); Thomas, W. s.n. CEPEC 57679 (2). 